\title{
A dislocation-based solution for stress introduced by arbitrary volume expansion in cylinders
}

Mathematics and Mechanics of Solids $1-18$

(C) The Author(s) 2018

Reprints and permissions:

sagepub.co.uk/journalsPermissions.nav DOI: $10.1177 / 1081286517751287$ journals.sagepub.com/home/mms @SAGE

\author{
Jici Wen \\ State Key Laboratory of Nonlinear Mechanics (LNM), Institute of Mechanics, Chinese \\ Academy of Sciences, Beijing, China; School of Engineering Science, University of Chinese \\ Academy of Science, Beijing, China
}

\section{Yujie Wei}

State Key Laboratory of Nonlinear Mechanics (LNM), Institute of Mechanics, Chinese

Academy of Sciences, Beijing, China; School of Engineering Science, University of Chinese

Academy of Science, Beijing, China

Received 23 June 2017; accepted 8 December 2017

\begin{abstract}
A cylindrical structure undergoing volume expansion and contraction is common in engineering practice. For example, the charging (discharging) process of axisymmetrical batteries will give rise to volume expansion (shrinkage). The nitriding process of axles for better fatigue performance also introduces volume expansion. Here, by taking the equivalence of volume expansion (or shrinkage) as continuous insertion (or distraction) of infinitesimal dislocations, we supply a framework to solve the stress field of a cylinder with arbitrary insertion (distraction) profile of materials along the radial direction. Under the assumptions that the volume expansion profile along the axis of a cylinder is uniform and the deformation is small so that the current configuration is regarded as the original, we supply analytical solutions of stress fields to several typical volume expansion or shrinkage profiles. Our analysis shows that different volume variation gives rise to either high tensile stress in the surface or hydrostatic tension in the core, and supplies distinct failure mechanisms in cylindrical batteries.
\end{abstract}

\section{Keywords}

Volume expansion, shrinkage, infinitesimal dislocation, stress, lithiation, delithiation, nitriding

\section{Introduction}

There is growing interest in understanding the stress introduced by material flow during material processing or electric-chemical-mechanical coupled fields. For example, the stress induced in a lithium-ion battery during lithiation and delithiation is a fundamental issue for the safety analysis of commercial batteries. Indeed, in the past decade, different design strategies for the anode materials have been proposed in order to alleviate the stress level during the charge-discharge cycle. An effective way is to use structures with high surface-to-volume ratio and to minimize the constraints to volumetric expansion [1$3]$ by tuning the interface and micro-nano level geometry for next-generation lithium-ion batteries [4-6].

Corresponding author:

Yujie Wei, State Key Laboratory of Nonlinear Mechanics (LNM), Institute of Mechanics, Chinese Academy of Sciences, Beijing I00I90, China.

Email: yujie_wei@Inm.imech.ac.cn 
Anode materials composed of wires have been demonstrated to be effective in reducing the stress level and improving the durability of lithium-ion batteries [1,7-18]. In situ observation of the electrochemical lithiation of a single $\mathrm{SnO}_{2}$ nanowire (NW) electrode [14] demonstrated that such NWs could withstand dramatic volume change without failure. Chan et al. [8] showed that silicon NW battery electrodes could achieve $75 \%$ of the theoretical discharge capacity of silicon without performance degradation after charge-discharge cycles. Using a unique transmission electron microscope technique, Ryu et al. [19] showed that Si NWs with diameters in the range of a few hundred nanometers can be fully lithiated and delithiated without fracture.

In different fields, material treatments such as carburizing, carbonitriding, and nitriding have been broadly employed in engineering practice for better fatigue performance of axles, bearings, wheels, and so on. The flow of $\mathrm{C}$ and $\mathrm{N}$ in the subsurface of those structures, in addition to strengthening, gives rise to high levels of residual compressive stress, which is crucial for fatigue enhancement. It is hence desired to understand the level of residual stress in order to achieve accurate prediction of fatigue performance and optimize the procedures of these treatments.

\section{Theoretical model}

Given the analogy in volume expansion between material insertion and heat flow, many mechanics models and numerical methods have been developed to capture the diffusion-induced stresses in batteries [20-28]. Cheng and Verbrugge [29,30] solved the stress evolution within a spherical insertion electrode particle under potentiostatic and galvanostatic operation. Bower et al. [31] analyzed the stress in spherical elastic-plastic lithium-ion electrode particles containing a propagating phase boundary. To examine the anisotropic swelling and fracture of silicon NWs during lithiation [32], Deshpande et al. [24] solved the stress evolution in NW electrode structures experiencing full charging or discharging process without discontinuities. Also, Harris et al. [33] analyzed the stress in NW structures with mesopores in the same way. In this paper, we take the analogy between volume expansion (shrinkage) and continuous insertion (distraction) of infinitesimal dislocations. Such a treatment enables us to deduce explicit stress solutions in NWs subjected to small to intermediate volume expansion.

\section{I. Problem description}

We consider a single NW undergoing an electrochemical lithium insertion or extraction process. Lithium ions flow into the wire along the radial direction and give rise to radial expansion, and consequently to radial stress and hoop stress. The radial stress, however, may reduce to zero to meet the traction-free boundary condition at the free surface if we ignore the surface effect at this moment. We will account for the deformation associated with different volume expansion profiles resulting from lithium insertion and extraction, and deduce the stress profile along the radial direction.

Geometrically, insertion of a thin layer of width $b$ in the wire resembles the insertion of a dislocation with Burgers vector $b$ in the wire. Inserting a wedge of constant angle $\theta$ in the wire is equivalent to the insertion of a disclination in the wire, as detailed in Figure 1. For the latter, the displacement and stress fields of a disclination running along the axis of a long isotropic cylinder were derived by Volterra [34]. Readers are referred to Christensen and Newman [22] for the stress field solution of a disclination with zero core running along the axis of an infinitely long isotropic cylinder. Indeed, Eshelby [35] solved the disclination problem in an infinite body and recognized that the stress field of two disclinations (a disclination dipole) of opposite sign is equivalent to that of a dislocation if the two disclinations are sufficiently close. In Figure 1, we illustrate how a wedge with zero core could be represented by the distribution of infinitesimal dislocations. Different distribution of dislocations along the radial direction of a wire with circular cross-section could be obtained by summing all dislocations in the cylindrical coordinate. Bearing this in mind, we may start from the stress fields of individual dislocations and construct their distribution to approximate an arbitrarily shaped wedge, from which the stress-field solution of the wedge could be derived.

\subsection{Stress induced by lithiation in wires}

Ideally, the concentration profile of ions along the radial direction of wires could be conveniently deduced if diffusion is driven by gradient alone. In the electrochemical lithium insertion and extraction 

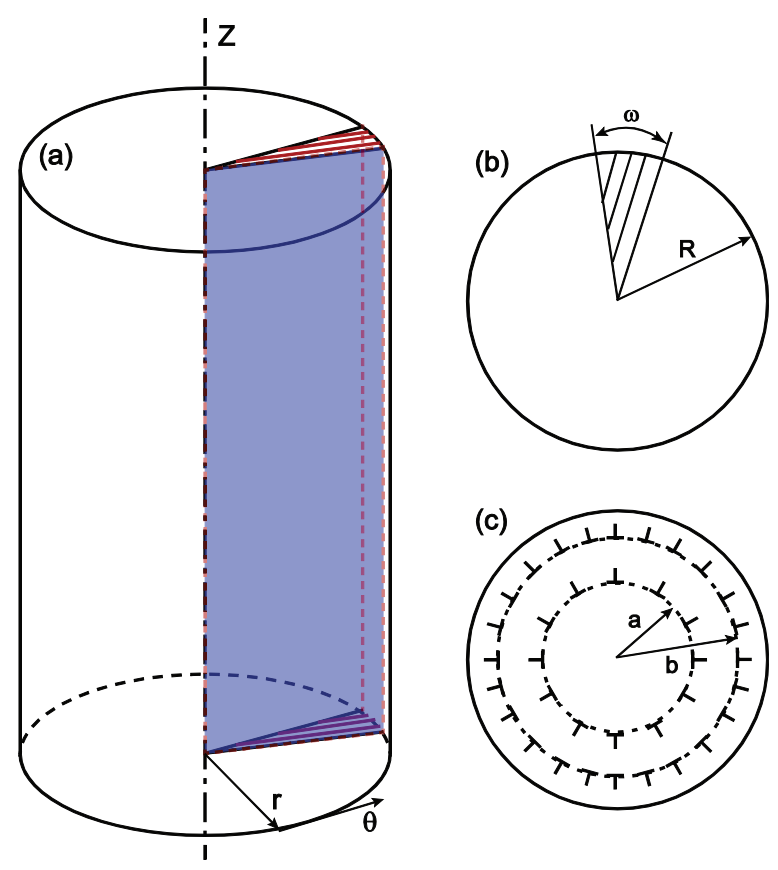

Figure I. Illustration of volume expansion in axisymmetric structures by inserting a wedge of constant angle (disclination). (a) The three-dimensional view (shadowed region). (b) The cross-section of the wire with the insertion of the wedge of angle $\omega$, which runs from the zero core to the surface. (c) Representation of the wedge of constant $\omega$ by infinitesimal dislocation distribution along the radial direction. From now on, we will use the cylindrical coordinate system defined by $(r, \theta, z)$.

process, complexity arises when the buildup stress may influence the local chemical potential of ions. In addition, a possible phase transition may occur between lithium ions and the anode materials. Taking Si as an example, the electrochemical lithiation of crystalline Si does not follow the thermodynamic phase diagram at room temperature [36]. At the end of discharge, the Li-Si phase undergoes an abrupt crystallization transformation to form $\mathrm{Li}_{15} \mathrm{Si}_{4}$ [37], which does not exist in the equilibrium phase diagram. Such factors certainly influence the theoretical analysis to obtain an exact concentration profile (hence the radius dependent volume expansion). For this consideration, we are not seeking the particular volume expansion solution in wires. Instead, we assume that the volume expansion is a known function of radius of an infinitely long isotropic cylinder, and we seek the stress solution in that circumstance.

As different volume expansion profiles can be regarded as the insertion of a particular wedge, we use dislocation distribution to approximate the wedge. We approach the solution by considering an array of edge dislocations uniformly residing in the circular boundary $r=r_{0}$ of the wire, and the Burgers vector of all dislocations is along the tangential direction of the circle. For information about the coordinate and the arrangement of dislocations, please refer to Figure 1. By defining $\rho\left(r_{0}\right)$ as the density of infinitesimal dislocations located in the ring from $r_{0}$ to $r_{0}+d r$, we obtain the geometrical equivalence of

$$
w\left(r_{0}\right)=\int_{0}^{r_{0}} \rho(r) r d \theta d r=2 \pi \int_{0}^{r_{0}} \rho(r) r d r
$$

where $w\left(r_{0}\right)$ is the wedge width at radius $r_{0}$. Hence $\rho\left(r_{0}\right)$ is the distribution of infinitesimal dislocations per unit area, and all Burgers vectors are parallel to the hoop direction, as seen in Figure 1. Hence $\rho(r)$ has a unit of $1 / \mathrm{m}$. For a wedge with constant $\omega$, we have $w\left(r_{0}\right)=\omega r_{0}$.

We first consider the case that an array of dislocations is placed evenly around the circle of radius $r_{0}$. The insertion of such an array of dislocations leads to the displacement in the hoop direction $u_{\theta}(r)$ written as 


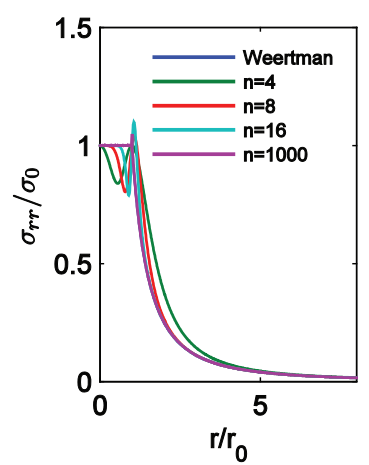

(a)

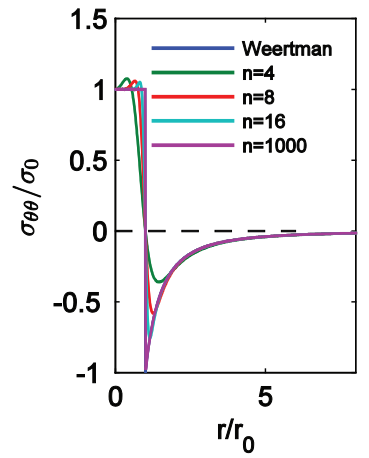

(b)

Figure 2. The radial stress and hoop stress induced by an array of dislocations placed evenly around the circle of radius $r_{0}$, theoretical analyses with equation (3) against discrete dislocation results. Here $\sigma_{0}=\frac{G \rho\left(r_{0}\right)}{(1-\nu)}$ and $n$ is the number of dislocations in the ring. (a) The radial stress versus radius. (b) The hoop stress with respect to radius.

$$
u_{\theta}(r)= \begin{cases}\frac{r_{0}^{2} \int_{0}^{\theta} d \theta \int_{r_{0}^{-}}^{r^{+}} \rho(r) d r}{r}=\frac{\theta r_{0}^{2} \hat{\rho}\left(r_{0}\right)}{r}, & r \geq r_{0} \\ 0, & r<r_{0}\end{cases}
$$

Since all dislocations reside along a circle of radius $r_{0}, \int_{r_{0}^{-}}^{r_{0}^{+}} \rho(r) d r=\hat{\rho}\left(r_{0}\right) H\left(r-r_{0}\right)$. Here $H(x)$ is the Heaviside step function, with $H(x)=1$ for $x>0$, and $H(x)=0$ otherwise. The density per unit area $\rho\left(r_{0}\right)$ recedes to the line density $\hat{\rho}$ of infinitesimal dislocations residing at the circle of radius $r_{0}$, in resemble of line strain; and $\hat{\rho}\left(r_{0}\right) r_{0} d \theta$ is the magnitude of the Burgers vector within the arc length $r_{0} d \theta$. Weertman [38] gave the stress field introduced by an array of dislocations distributed in the ring of radius $r_{0}$ :

$$
\left\{\begin{array}{l}
\sigma_{\theta \theta}=\sigma_{r r}=\frac{G \hat{\rho}\left(r_{0}\right)}{(1-\nu)}+c, r \leq r_{0} \\
\sigma_{\theta \theta}=-\frac{G \hat{\rho}\left(r_{0}\right)}{(1-\nu)} \frac{r_{0}^{2}}{r^{2}}+c, \sigma_{r r}=\frac{G \hat{\rho}\left(r_{0}\right)}{(1-\nu)} \frac{r_{0}^{2}}{r^{2}}+c, r>r_{0}
\end{array}\right.
$$

The constant $c$ in equation (3) is related to the boundary condition at the outer surface of the wire; $G$ and $\nu$ are the shear modulus and the Poisson's ratio of the isotropic material. The validity of equation (3) has been examined by uniformly distributing an array of dislocations in the ring of radius $r_{0}$. The dependence of stresses on radius, by summing up the contribution from all dislocations, is presented in Figure 2. It is clearly seen that both the radial stress (Figure 2(a)) and the hoop stress (Figure 2(b)) tend to converge to the theoretical solution as $n \rightarrow \infty$.

Now let us get back to the more general case that $\rho\left(r_{0}\right)$ is the dislocation distribution of infinitesimal dislocations per unit area. Then for dislocations residing in the ring from $r_{0}$ to $r_{0}+d r$, they introduce stress fields which are given as

$$
\left\{\begin{array}{l}
\sigma_{\theta \theta}=\sigma_{r r}=\frac{G \rho\left(r_{0}\right) d r}{(1-\nu)}+c d r, r \leq r_{0} \\
\sigma_{\theta \theta}=-\frac{G \rho\left(r_{0}\right) d r}{(1-\nu)} \frac{r_{0}^{2}}{r^{2}}+c d r, \sigma_{r r}=\frac{G \rho\left(r_{0}\right) d r}{(1-\nu)} \frac{r_{0}^{2}}{r^{2}}+c d r, r>r_{0}
\end{array}\right.
$$

Using equation (4) as the Green's function, we could write out the stress fields at $r$ by dislocations distributed from $a$ to $R$ as 


$$
\begin{gathered}
\sigma_{\theta \theta}(r)=\int_{r}^{R} \frac{G \rho\left(r^{\prime}\right)}{1-\nu} d r^{\prime}-\int_{a}^{r} \frac{G \rho\left(r^{\prime}\right) r^{\prime 2}}{(1-\nu) r^{2}} d r^{\prime}+c(R-a) \\
\sigma_{r r}(r)=\int_{r}^{R} \frac{G \rho\left(r^{\prime}\right)}{1-\nu} d r^{\prime}+\int_{a}^{r} \frac{G \rho\left(r^{\prime}\right) r^{\prime 2}}{(1-\nu) r^{2}} d r^{\prime}+c(R-a)
\end{gathered}
$$

Here $r^{\prime}$ is an integral variable. In the region $0 \leq r \leq a$, we have $\sigma_{\theta \theta}(r)=\sigma_{\theta \theta}(a)$ and $\sigma_{r r}(r)=\sigma_{r r}(a)$. The constants $c$ can be determined by the traction-free boundary condition:

$$
\sigma_{r r}=0 \text { at } r=R
$$

Equations (1) and (5) supply the framework to solve the stress fields of cylindrical elastic solids subjected chemical or mechanical induced deformation with known radius-dependent volume expansion profiles. In the following section, we examine the stress fields introduced by typical volume expansion profiles using equations (1) and (5).

\section{Solution to particular volume expansion profiles}

As we mentioned previously, during the electrochemical lithiation process, the concentration of lithium ions may not follow the classic solution at thermodynamic equilibrium. Therefore, the exact concentration profile cannot be easily obtained if the electrochemical and chemo-mechanical processes and related coupling equations are not fully identified and solved. We circumvent this difficulty by taking the volume expansion profile as being a priori. Different volume expansion profiles, which may be possibly due to the variation in charge-discharge rate, are considered here.

\section{I. Linear volume expansion during charging}

The number of dislocations in the wire is so distributed that the insertion is a wedge of constant angle $\omega^{a}$. Mathematically, we have $w\left(r^{\prime}\right)=\omega^{a}\left(r^{\prime}-a\right)$. We show in Figure 3(a) the insertion of a wedge of constant angle in the wire from the radius $a$ to $R$. Using equation (1), we have the dislocation distribution function

$$
\rho\left(r^{\prime}\right)=\frac{\omega^{a}}{2 \pi r^{\prime}} \text { for } a \leq r^{\prime} \leq R
$$

Figure 3(b) gives the corresponding dislocation distribution along the radial direction. Inserting equation (7) into equation (5), we obtain the corresponding hoop stress and the radial stress in the wire. All other stress components in the cylindrical coordinate are zero.

$$
\begin{gathered}
\sigma_{\theta \theta}=\frac{G \omega^{a}}{2 \pi(1-\nu)} \ln \frac{R}{r}-\frac{G \omega^{a}}{4 \pi(1-\nu)}\left(1-\frac{a^{2}}{r^{2}}\right)+c(R-a) \\
\sigma_{r r}=\frac{G \omega^{a}}{2 \pi(1-\nu)} \ln \frac{R}{r}+\frac{G \omega^{a}}{4 \pi(1-\nu)}\left(1-\frac{a^{2}}{r^{2}}\right)+c(R-a) \\
c=-\frac{G \omega^{a}}{2 \pi(1-\nu)} \frac{R+a}{2 R^{2}}
\end{gathered}
$$

It is convenient to see that we give the stress solution of a disclination wedge of constant angle $\omega^{a}$ in the wire from $a$ to $R$. If $a=0$, the solution shown above is the exact solution of a disclination running along the axis of an infinitely long isotropic cylinder, as given by Volterra [34]. For more recent references, readers are referred to the paper by De Wit [39]. The only difference is that the radius here is finite and we have an extra term to balance the traction induced by the dislocation. We also define the hydrostatic stress $\sigma_{h}$ as 


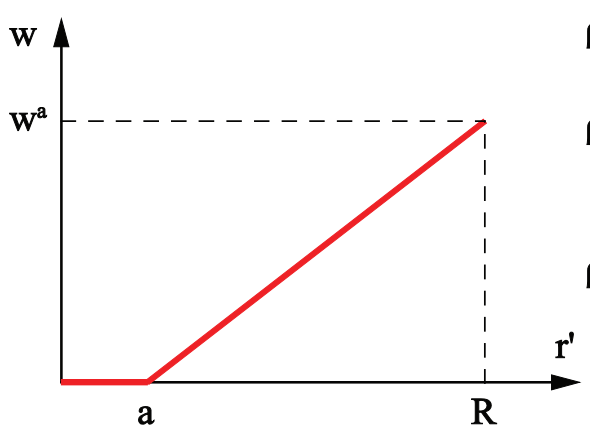

(a)

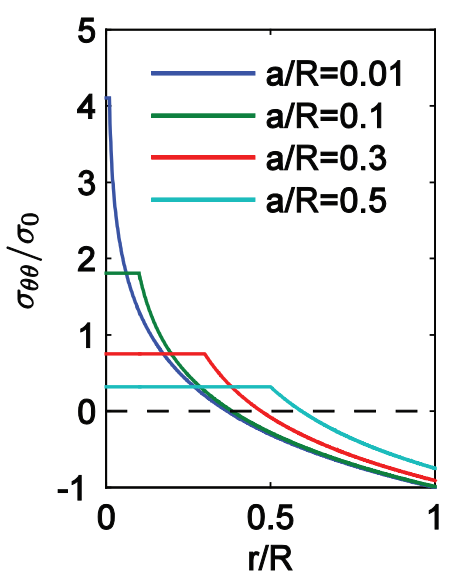

(C)

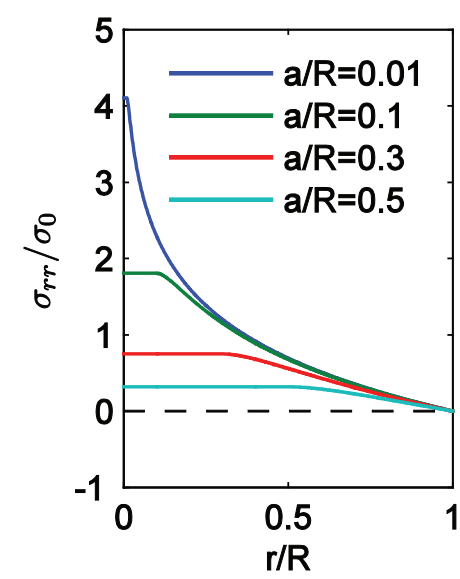

(d)

Figure 3. Volume expansion profile and subsequent stress in the wire. (a) The wedge width along the radius, with $w^{a}=\omega^{a}(R-a)$. (b) Corresponding dislocation distribution profile along the radius direction, where $\rho^{a}=\frac{\omega^{a}}{2 \pi a}$ and $\rho^{b}=\frac{\omega^{a}}{2 \pi R}$. The normalized stress in the wire with different insertion location of the wedge: (c) the hoop stress with respect to radius; (d) the radial stress; (e) the hydrostatic stress.

$$
\sigma_{h}=\frac{\alpha\left(\sigma_{\theta \theta}+\sigma_{r r}\right)}{3}
$$

where $\alpha$ is a parameter relating to the boundary condition along the wire axis. For confined deformation along the wire axis (plane strain), $\alpha=(1+\nu)$, and $\alpha=1$ for a thin disk (plane stress). For convenience, we use $\alpha=1$ in what follows.

We show in Figure 3(c) to (e) the stress as a function of radius. For convenience, we normalize all stress components by $\sigma_{0}$, which is defined as $\sigma_{0}=\frac{G w^{a}}{2 \pi(1-\nu)(R-a)}$. As can be seen in Figure 3(c), the hoop stress is tensile in the inner core of the wire but compressive in the outer shell. The transitional point is not exactly the wedge tip but locates slightly away from the tip near the surface side. The radial stress is tensile along the radius, increasing from the surface to the core, but saturates at the wedge tip and then remains constant (Figure 3(d)). The hydrostatic stress, similar to the hoop stress, is compressive in the outer shell and tensile in the inner core (Figure 3(e)). It hence implies the possibility of introducing internal tensile failure during the charging state.

\subsection{Radical volume expansion gradient}

We now consider the insertion of a wedge with different gradient. In that circumstance, the profile of the wedge is a polynomial function of the radius $r^{\prime}$. The volume expansion could result from different charging rates, which give rise to different wedge shape, as illustrated in Figure 4(a). The function to describe the three representative wedge profiles in Figure 4(a) is given as 


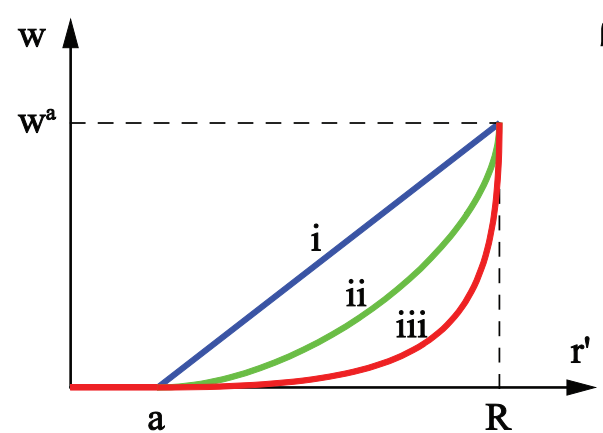

(a)

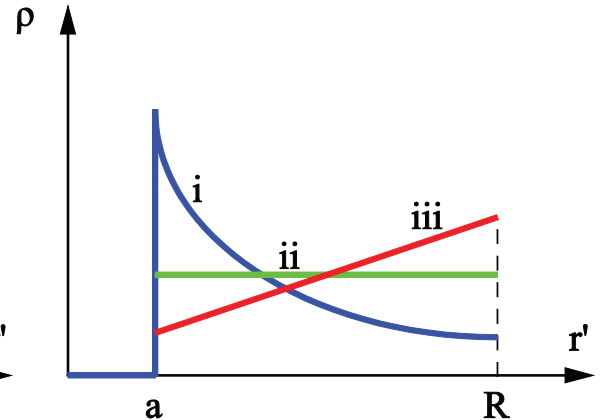

(b)

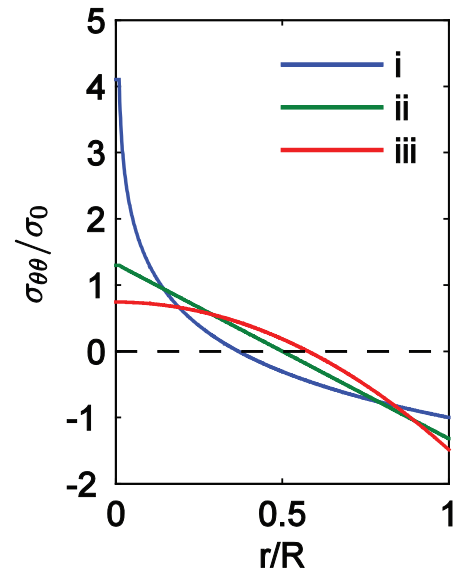

(c)

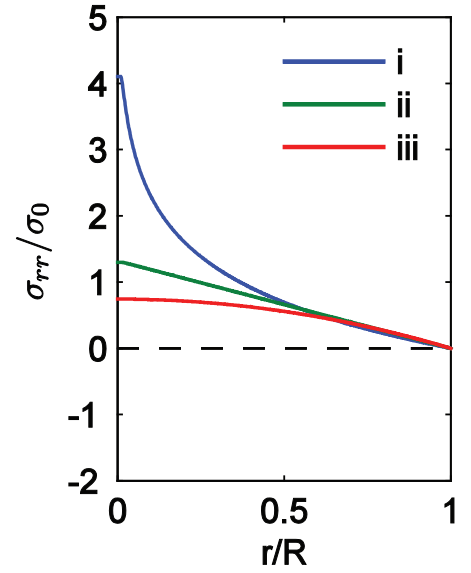

(d)

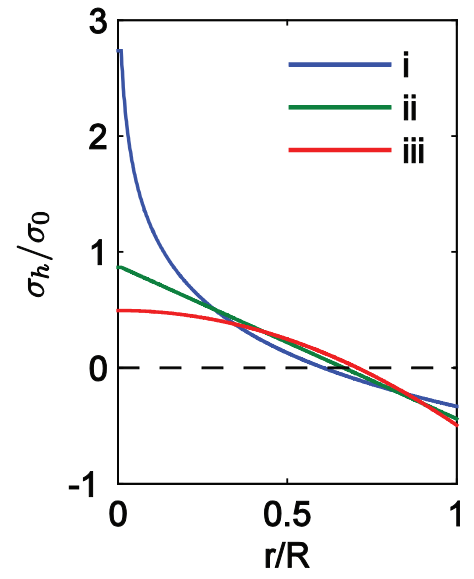

(e)

Figure 4. Wedge profile with different gradient and consequent stresses. (a) The wedge width respect to radius. Here $w^{a}=\omega^{a}(R-a)+\omega^{b} \frac{\left(R^{2}-a^{2}\right)}{2 R}+\omega^{c} \frac{\left(R^{3}-a^{3}\right)}{3 R^{2}}$. (a) Dislocation distribution profile respect to radius. For the three curves, we have $\omega^{b}=\omega^{c}=0$ for case "i," $\omega^{a}=\omega^{c}=0$ for case "ii," and $\omega^{a}=\omega^{b}=0$ for case "iii." The influence of wedges of different shape on stress: (c) the hoop stress as a function of radius (the influence of different wedge profiles is shown); (d) the radial stress; (e) the hydrostatic stress.

$$
w\left(r^{\prime}\right)=\omega^{a}\left(r^{\prime}-a\right)+\omega^{b} \frac{\left(r^{\prime 2}-a^{2}\right)}{2 R}+\omega^{c} \frac{\left(r^{\prime 3}-a^{3}\right)}{3 R^{2}}
$$

With equation (1), we have the following dislocation distribution profile:

$$
\rho\left(r^{\prime}\right)=\frac{\omega^{a}+\omega^{b} r^{\prime} / R+\omega^{c} r^{\prime 2} / R^{2}}{2 \pi r^{\prime}} \text { for } \mathrm{a} \leq r^{\prime} \leq \mathrm{R}
$$

By inserting equation (10) into equation (5), we obtain the corresponding hoop stress and the radial stress in the wire as

$$
\begin{aligned}
& \sigma_{\theta \theta}=\frac{G \omega^{a}}{2 \pi(1-\nu)} \ln \frac{R}{r}-\frac{G \omega^{a}}{4 \pi(1-\nu)}\left(1-\frac{a^{2}}{r^{2}}\right)+\frac{G \omega^{b}}{2 \pi(1-\nu)}\left(1-\frac{r}{R}\right)- \\
& \frac{G \omega^{b}}{6 \pi(1-\nu)} \frac{r}{R}\left(1-\frac{a^{3}}{r^{3}}\right)+\frac{G \omega^{c}}{4 \pi(1-\nu)}\left(1-\frac{r^{2}}{R^{2}}\right)- \\
& \frac{G \omega^{c}}{8 \pi(1-\nu)} \frac{r^{2}}{R^{2}}\left(1-\frac{a^{4}}{r^{4}}\right)+c(R-a)
\end{aligned}
$$




$$
\begin{gathered}
\sigma_{r r}=\frac{G \omega^{a}}{2 \pi(1-\nu)} \ln \frac{R}{r}+\frac{G \omega^{a}}{4 \pi(1-\nu)}\left(1-\frac{a^{2}}{r^{2}}\right)+\frac{G \omega^{b}}{2 \pi(1-\nu)}\left(1-\frac{r}{R}\right)+ \\
\frac{G \omega^{b}}{6 \pi(1-\nu)} \frac{r}{R}\left(1-\frac{a^{3}}{r^{3}}\right)+\frac{G \omega^{c}}{4 \pi(1-\nu)}\left(1-\frac{r^{2}}{R^{2}}\right)+ \\
\frac{G \omega^{c}}{8 \pi(1-\nu)} \frac{r^{2}}{R^{2}}\left(1-\frac{a^{4}}{r^{4}}\right)+c(R-a) \\
c=-\frac{G \omega^{a}}{4 \pi(1-\nu)} \frac{(R+a)}{R^{2}}-\frac{G \omega^{b}}{6 \pi(1-\nu)} \frac{R^{2}+a R+a^{2}}{R^{3}}-\frac{G \omega^{c}}{8 \pi(1-\nu)} \frac{\left(R^{2}+a^{2}\right)(R+a)}{R^{4}}
\end{gathered}
$$

We show in Figure 4(c) to (e) the stress components normalized by $\sigma_{0}$. For the same wedge width $w^{a}$ at $r=R$ and also $a=0$, we see from Figure 4(c) that the hoop stress shows significant difference in the core region as the wedge profile changes. Higher gradient in volume expansion, while increasing the compressive stress in the outer shell of the wire, reduces the magnitude of the tensile stress. The same trend applies to the variation of radial stress with radius, as can be seen in Figure 4(d). Correspondingly, the hydrostatic stress is compressive in the outer shell, which increases with higher gradient, but the hydrostatic tensile stress decreases at higher gradient, as shown in Figure 4e.

\subsection{Volume expansion with saturation}

Now we consider the case of a two-stage wedge profile. In the core, the wedge has a constant angle but in the shell, the width of the wedge remains constant, as can be seen in Figure 5(a). In this circumstance, it may resemble a charging front moving toward the core of the wire, and the outer shell is fully charged. The profile of the wedge is described as

$$
w\left(r^{\prime}\right)= \begin{cases}w^{a} \frac{\left(r^{\prime}-a\right)}{b-a}, & a \leq r^{\prime} \leq b \\ w^{a}, & b<r^{\prime} \leq R\end{cases}
$$

It is convenient to obtain the dislocation distribution profile with equation (1):

$$
\rho\left(r^{\prime}\right)= \begin{cases}\frac{w^{a}}{2 \pi r^{\prime}(b-a)}, & a \leq r^{\prime} \leq b \\ 0, & b<r^{\prime} \leq R\end{cases}
$$

By inserting equation (13) into equation (5), we obtain the corresponding hoop stress and the radial stress in the wire as

$$
\begin{gathered}
\sigma_{\theta \theta}= \begin{cases}-\frac{G w^{a}}{2 \pi(1-\nu)(b-a)} \frac{r^{2}-a^{2}}{2 r^{2}}+\frac{G w^{a}}{2 \pi(1-\nu)(b-a)} \ln \frac{b}{r}+c(R-a) & , a \leq r \leq b \\
-\frac{G w^{a}}{2 \pi(1-\nu)(b-a)} \frac{b^{2}-a^{2}}{2 r^{2}}+c(R-a), & b<r \leq R\end{cases} \\
\sigma_{r r}= \begin{cases}\frac{G w^{a}}{2 \pi(1-\nu)(b-a)} \frac{r^{2}-a^{2}}{2 r^{2}}+\frac{G w^{a}}{2 \pi(1-\nu)(b-a)} \ln \frac{b}{r}+c(R-a), & a \leq r \leq b \\
\frac{G w^{a}}{2 \pi(1-\nu)} \frac{b+a}{2 r^{2}}+c(R-a), & b<r \leq R\end{cases} \\
c=-\frac{G w^{a}}{2 \pi(1-\nu)} \frac{b+a}{2(R-a) R^{2}}
\end{gathered}
$$




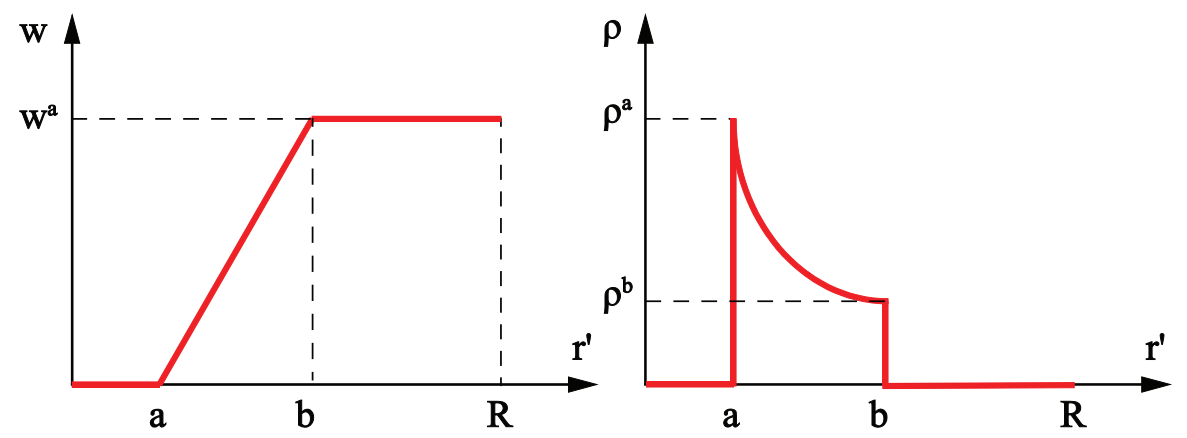

(a)

(b)

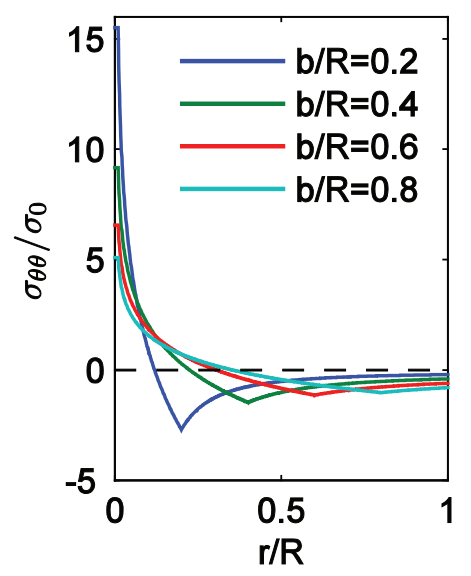

(c)

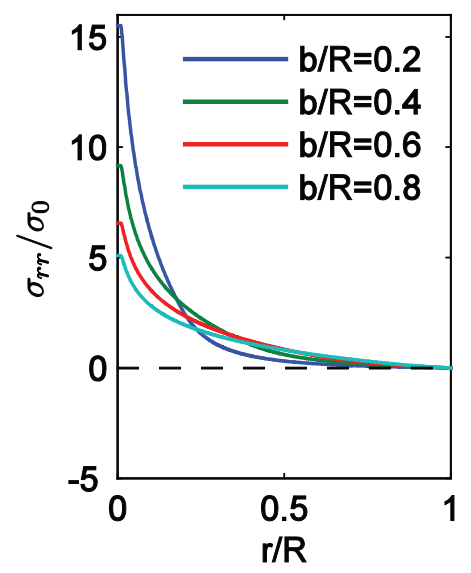

(d)

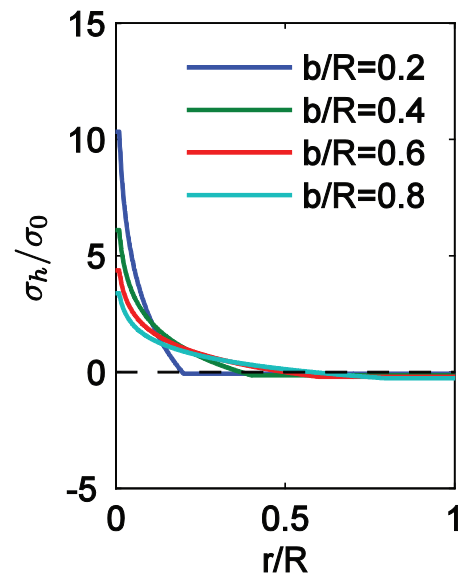

(e)

Figure 5. Two-stage wedge profiles representing different charging states and the stresses. (a) The wedge width along the radius. (b) Dislocation distribution profile along the radius. The influence of transitional point for two stage wedges: (c) the hoop stress as a function of radius for different transitional point; (d) the radial stress; (e) the hydrostatic stress.

The normalized stress components (by $\sigma_{0}$ ) as a function of radius are shown in Figure 5(c) to (e). We let the wedge width $w^{a}$ at $r=R$ be the same and let $a=0$. Figure 5(c) shows the evolution of the hoop stress for several typical transitional points $b$. Within the region where the wedge width is a constant, the hoop stress is compressive and it decreases from the shell to the core, and then starts to increase at the transitional point from compressive to tensile in the core region. As point $b$ moves closer to the core, the hoop stress is higher. The variation of radial stress with radius is seen in Figure 5(d). The radial stress is tensile and its magnitude decreases with increasing radius monotonically. From Figure 5(e), we see that the hydrostatic stress within the region of constant wedge width is compressive. It then increases from the transitional point to the core of the wire $(r=0)$. When point $b$ is closer to the core, the hydrostatic tensile stress becomes greater.

\section{Stress induced by delithiation}

In the previous section, we consider the charging (insertion) case where the wedge width (volume expansion) is non-decreasing with radius. Now we deal with intermediate stage when the volume expansion reduces with radius, which may resemble the volume expansion profile during discharge.

\section{I. Volume shrinkage with linear gradient}

We first consider a simple case that volume expansion as a function of the radius is of trapezoidal profile, as demonstrated in Figure. 6(a). It is noted if we let $a=b=0$, then it suggests that the volume 


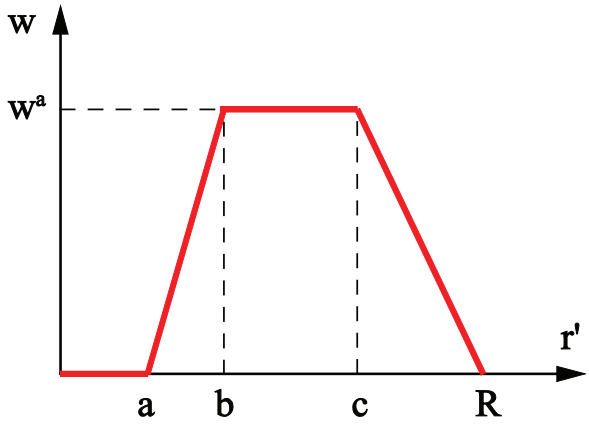

(a)

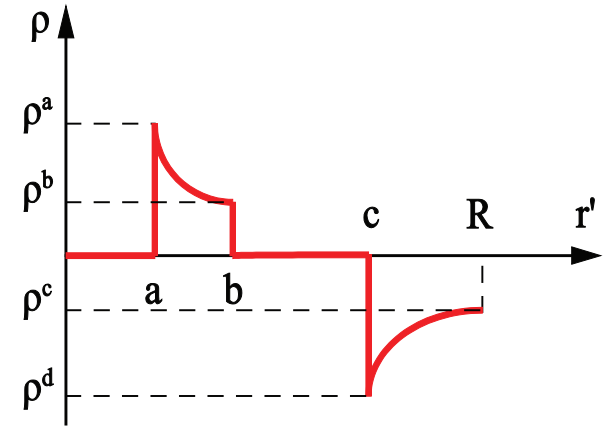

(b)

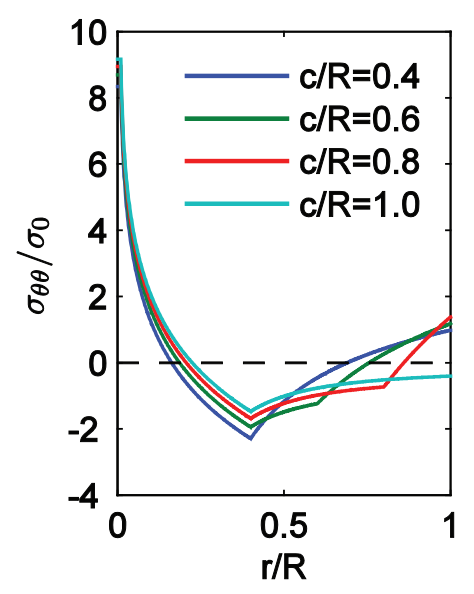

(c)

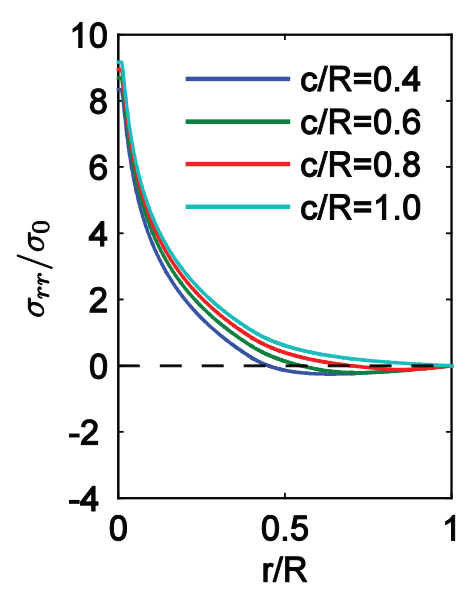

(d)

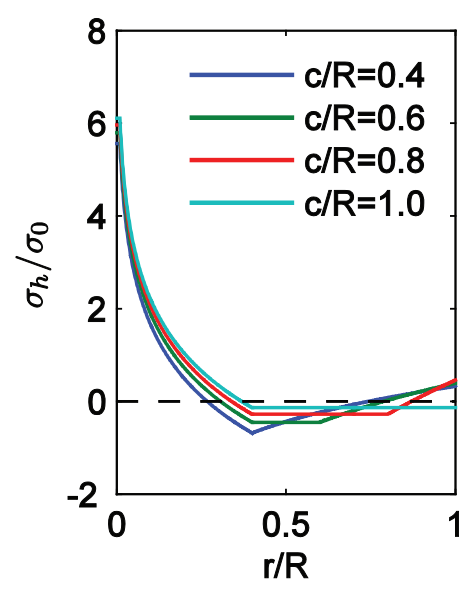

(e)

Figure 6. Volume expansion during the discharging of an initially fully charged wire and the stress evolution. (a) The wedge width along the radius. (b) Corresponding dislocation distribution profile along the radius. Stress evolution due to volume shrinkage of an initially fully charged wire experiencing discharge: (c) the hoop stress as a function of radius; (d) the radial stress; (e) the hydrostatic tensile stress.

expansion in the core of the wire is a constant but that in the outer shell decreases linearly. With equation (1), we have a dislocation distribution as a function of radius seen in Figure. 6(b). In this circumstance, it may resemble the scenario of an initially fully charged wire in the middle of discharging. The function for the wedge profile and the dislocation distribution as a function of radius are given as

$$
w\left(r^{\prime}\right)=\left\{\begin{array}{l}
w^{a} \frac{r^{\prime}-a}{b-a}, a \leq r^{\prime} \leq b \\
w^{a}, \quad b<r^{\prime} \leq c \\
w^{a} \frac{r^{\prime}-R}{c-R}, c<r^{\prime} \leq R
\end{array}\right.
$$

and

$$
\rho\left(r^{\prime}\right)= \begin{cases}\frac{w^{a}}{2 \pi r^{\prime}(b-a)}, & a \leq r^{\prime} \leq b \\ 0, & b<r^{\prime} \leq c \\ +\frac{w^{a}}{2 \pi r^{\prime}(c-R)}, & c<r^{\prime} \leq R\end{cases}
$$


respectively. By inserting equation (16)into equation (5), we obtain the corresponding hoop stress and the radial stress in the wire as:

$$
\begin{gathered}
\sigma_{\theta \theta}= \begin{cases}\frac{G w^{a}}{2 \pi(1-\nu)(R-a)}\left[\frac{R-a}{c-R} \ln \frac{R}{c}+\frac{R-a}{b-a} \ln \frac{b}{r}-\frac{R-a r^{2}-a^{2}}{b-a}\right]+c(R-a), & a \leq r \leq b \\
\frac{G w^{a}}{2 \pi(1-\nu)(R-a)}\left[\frac{R-a}{c-R} \ln \frac{R}{c}-\frac{R-a}{b-a} \frac{b^{2}-a^{2}}{2 r^{2}}\right]+c(R-a), & b<r \leq c \\
\frac{G w^{a}}{2 \pi(1-\nu)(R-a)}\left[\frac{R-a}{c-R} \ln \frac{R}{r}-\frac{R-a}{c-R} \frac{r^{2}-c^{2}}{2 r^{2}}-\frac{R-a}{b-a} \frac{b^{2}-a^{2}}{2 r^{2}}\right]+c(R-a), c<r \leq R\end{cases} \\
\sigma_{r r}= \begin{cases}\frac{G w^{a}}{2 \pi(1-\nu)(R-a)}\left[\frac{R-a}{c-R} \ln \frac{R}{c}+\frac{R-a}{b-a} \ln \frac{b}{r}+\frac{R-a r^{2}-a^{2}}{b-a}\right]+c(R-a), & a \leq r \leq b \\
\frac{G w^{a}}{2 \pi(1-\nu)(R-a)}\left[\frac{R-a}{c-R} \ln \frac{R}{c}+\frac{R-a}{b-a} \frac{b^{2}-a^{2}}{2 r^{2}}\right]+c(R-a), & b<r \leq c \\
\frac{G w^{a}}{2 \pi(1-\nu)(R-a)}\left[\frac{R-a}{c-R} \ln \frac{R}{r}+\frac{R-a}{c-R} \frac{r^{2}-c^{2}}{2 r^{2}}+\frac{R-a}{b-a} \frac{b^{2}-a^{2}}{2 r^{2}}\right]+c(R-a), c<r \leq R\end{cases} \\
c=-\frac{G w^{a}}{2 \pi(1-\nu)(R-a)}\left[\frac{1}{c-R} \frac{R^{2}-c^{2}}{2 R^{2}}+\frac{1}{b-a} \frac{b^{2}-a^{2}}{2 R^{2}}\right]
\end{gathered}
$$

We show in Figure 6(c) to (e) the normalized stress components (by $\sigma_{0}$ ) as a function of radius. Again, we let the wedge width $w^{a}$ at $r=R$ be the same, $a=0.01 R$, and $b=0.4 R$. In contrast to previous charging circumstances, we demonstrate in Figure 6(c) that the discharging induced volume shrinkage does introduce tensile hoop stress at the outer shell of the wire. Although the magnitude of the tensile stress is small in contrast to that in the core, it might introduce crack initiation from the surface where preexisting imperfections could lower the critical strength for brittle fracture dramatically. Regardless the significant variation in volume profile, the radial stress is tensile along the radial direction, decreasing from the inner core to zero at the outer surface, as seen in Figure 6(d). From Figure 6(e), we see that the outer shell could be under hydrostatic tension during discharging.

\subsection{Volume shrinkage at different gradient rate}

Similar to the case discussed for charging rate, we also assume that the discharging rate would give rise to different volume expansion profile. Hence the wedge profile may have different gradient due to rate effect. A simple polynomial equation is used to describe the variation of the wedge profile, which is given as:

$$
w\left(r^{\prime}\right)= \begin{cases}w^{a} \frac{r^{\prime}-a}{b-a}, & a \leq r^{\prime} \leq b \\ w^{a}, & b<r^{\prime} \leq c \\ w^{a}-w^{a}\left(\frac{r^{\prime}-c}{R-c}\right)^{m}, & , c<r^{\prime} \leq R\end{cases}
$$

where $m$ represents different decreasing gradients if $m>0$. The exact shape of the profile is demonstrated in Figure 7(a). We have used three different $m$ to represent the profile difference. Here $m=1,2$, and 3, in turn, correspond to cases "i," "ii," and "iii." With equation (1), it is straightforward to write out the dislocation density profile as a function of radius: 


$$
\rho\left(r^{\prime}\right)= \begin{cases}\frac{w^{a}}{2 \pi r^{\prime}(b-a)}, & a \leq r^{\prime} \leq b \\ 0, & b<r^{\prime} \leq c \\ -\frac{w^{a} m}{2 \pi r^{\prime}(R-c)^{m}}\left(r^{\prime}-c\right)^{m-1}, & c<r^{\prime} \leq R\end{cases}
$$

Figure 7(b) shows the corresponding dislocation density curve. By inserting equation (19) into equation (5), we obtain the corresponding hoop stress and the radial stress in the wire as

$$
\begin{aligned}
& \int \frac{G w^{a}}{2 \pi(1-v)(R-a)}\left[-\frac{m(R-a)}{(R-c)^{m}} \int_{c}^{R} \frac{\left(r^{\prime}-c\right)^{m-1}}{r^{\prime}} d r^{\prime}+\frac{R-a}{b-a} \ln \frac{b}{r}-\frac{R-a r^{2}-a^{2}}{b-a} \frac{2 r^{2}}{b}\right] \\
& +c(R-a), a \leq r \leq b \\
& \sigma_{\theta \theta}=\left\{\begin{array}{c}
\frac{G w^{a}}{2 \pi(1-v)(R-a)}\left[-\frac{m(R-a)}{(R-c)^{m}} \int_{c}^{R} \frac{\left(r^{\prime}-c\right)^{m-1}}{r^{\prime}} d r^{\prime}-\frac{R-a}{b-a} \frac{b^{2}-a^{2}}{2 r^{2}}\right] \\
\quad+c(R-a), b<r \leq c
\end{array}\right] \\
& \frac{G w^{a}}{2 \pi(1-v)(R-a)}+c(R-a), c<r \leq R \\
& \int \frac{G w^{a}}{2 \pi(1-v)(R-a)}\left[-\frac{m(R-a)}{(R-c)^{m}} \int_{c}^{R} \frac{\left(r^{\prime}-c\right)^{m-1}}{r^{\prime}} d r^{\prime}+\frac{R-a}{b-a} \ln \frac{b}{r}+\frac{R-a r^{2}-a^{2}}{b-a} \frac{2 r^{2}}{b}\right] \\
& +c(R-a), a \leq r \leq b \\
& \sigma_{r r}=\left\{\begin{aligned}
\frac{G w^{a}}{2 \pi(1-v)(R-a)}[ & \left.-\frac{m(R-a)}{(R-c)^{m}} \int_{c}^{R} \frac{\left(r^{\prime}-c\right)^{m-1}}{r^{\prime}} d r^{\prime}+\frac{R-a}{b-a} \frac{b^{2}-a^{2}}{2 r^{2}}\right] \\
& +c(R-a), b<r \leq c
\end{aligned}\right. \\
& {\left[-\frac{m(R-a)}{(R-c)^{3}} \int_{r}^{R} \frac{\left(r^{\prime}-c\right)^{m-1}}{r^{\prime}} d r^{\prime}-\frac{m(R-a)}{(R-c)^{m}} \int_{c}^{r} \frac{r^{\prime}}{r^{2}}\left(r^{\prime}-c\right)^{m-1} d r^{\prime}+\frac{R-a}{b-a} \frac{b^{2}-a^{2}}{2 r^{2}}\right]} \\
& \frac{G w^{a}}{2 \pi(1-v)(R-a)}+c(R-a), c<r \leq R \\
& c=-\frac{G w^{a}}{2 \pi(1-\nu)(R-a)}\left[-\frac{m}{(R-c)^{m}} \int_{c}^{R} \frac{r^{\prime}}{R^{2}}\left(r^{\prime}-c\right)^{m-1} d r^{\prime}+\frac{1}{b-a} \frac{b^{2}-a^{2}}{2 R^{2}}\right]
\end{aligned}
$$

We let the wedge width $w^{a}$ at $r=R$ be the same, $a=0.01 R$, and $b=c=0.4 R$. In contrast to the linear discharging circumstance, the normalized stress (by $\left.\sigma_{0}\right)$-radius curves shown in Figure 7(c) to (e) suggest that the stresses are insensitive to the three different profiles. Consistently, all volume shrinkage with different gradient introduces tensile hoop stress and hydrostatic tension at the outer shell of the wire.

\section{Volume variation in exponential decay}

Following the solution for steady-state diffusion where the concentration follows an exponential decay, we now consider the case that the volume expansion along the radial direction follows an exponential increase (steady-state charge). We are interested in the stress variation as a function of position. For generality, we consider a wedge profile given by 


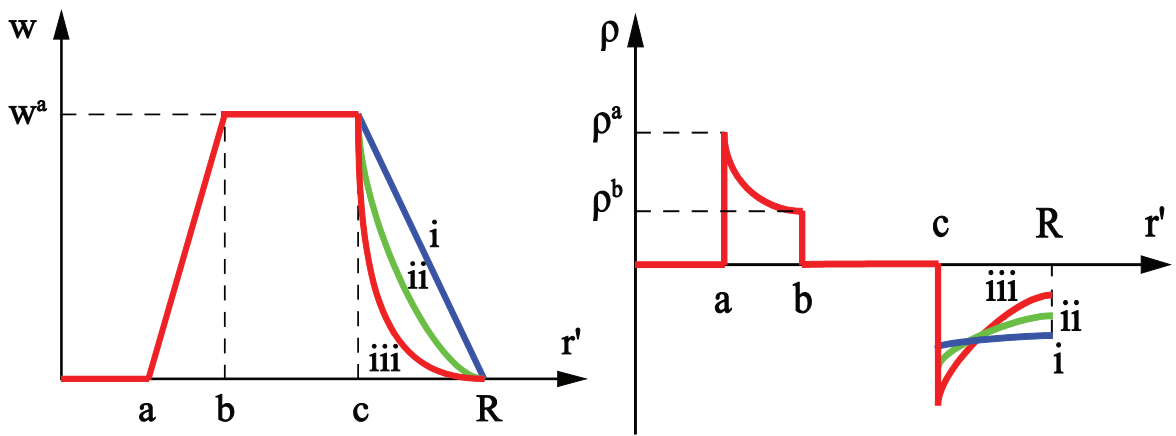

(a)

(b)

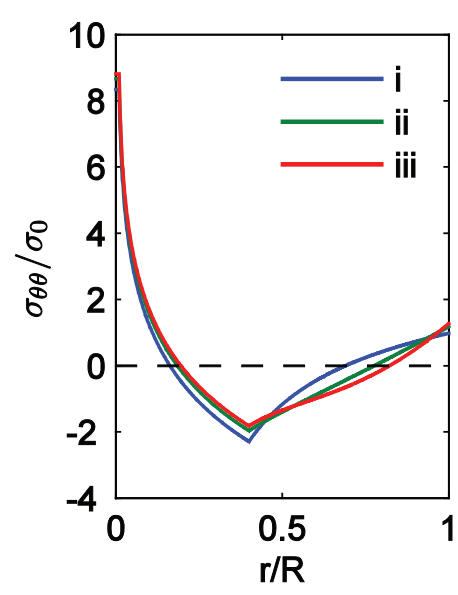

(C)

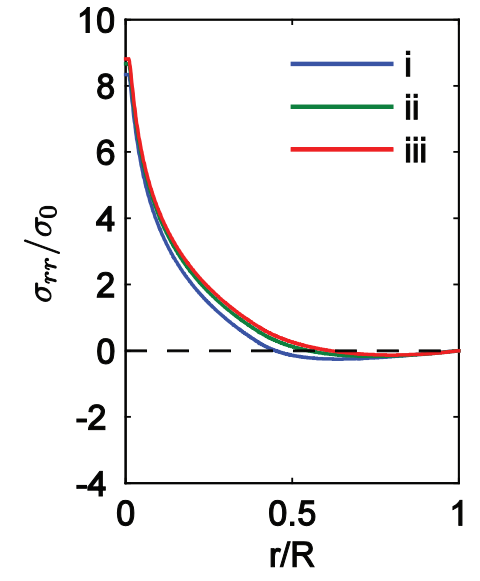

(d)

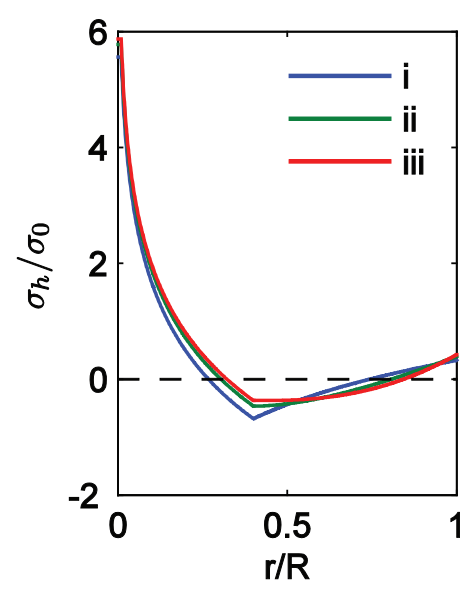

(e)

Figure 7. Volume shrinkage at different rate during the discharging of an initially fully charged wire and the stress evolution in wire. (a) The wedge width along the radius. In the outer shell, different volume shrinkage rates are shown. (b) Corresponding dislocation distribution profile along the radius. Here $m=1,2$, and 3 in equation (I8), in turn, corresponds to the case of "i,"“ii," and "iii." The influence of volume shrinkage rate to stress in an initially fully charged wire under discharging: (c) the hoop stress as a function of radius; (d) the radial stress; (e) the hydrostatic tensile stress.

$$
w\left(r^{\prime}\right)=\frac{m R}{n}\left(e^{\frac{n \prime^{\prime}}{R}}-e^{\frac{n a}{R}}\right)
$$

where $m$ and $n$ are two dimensionless coefficients which could be used to control the characteristic shrinkage length. In Figure 8, we show the profile of the wedge described by equation (21). The corresponding distribution of dislocations along the radial direction is given as

$$
\rho\left(r^{\prime}\right)=\frac{1}{2 \pi r^{\prime}} m e^{n r^{\prime}} \text { for } a \leq r^{\prime} \leq R
$$

Now the hoop stress and the radial stress, after solving equation (5), are given as

$$
\begin{aligned}
& \sigma_{\theta \theta}=-\frac{G m}{2 \pi(1-\nu)} \frac{1}{n^{2}} \frac{1}{\left(\frac{r}{R}\right)^{2}}\left[\left(n \frac{r}{R}-1\right) e^{\frac{n r}{R}}-\left(n \frac{a}{R}-1\right) e^{\frac{n a}{R}}\right]+ \\
& \frac{G m}{2 \pi(1-\nu)} \int_{\frac{n r}{R}}^{n} g^{-1} e^{g} d g+c(R-a)
\end{aligned}
$$




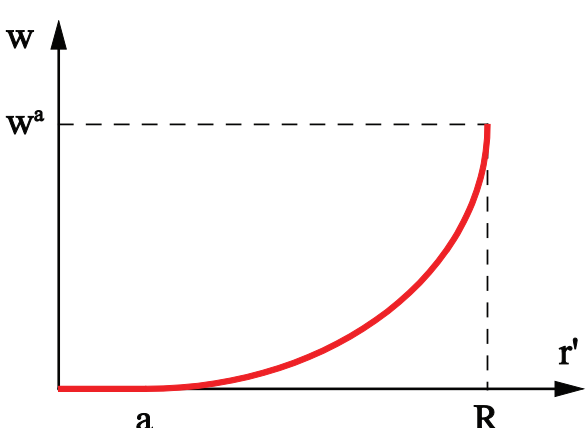

(a)

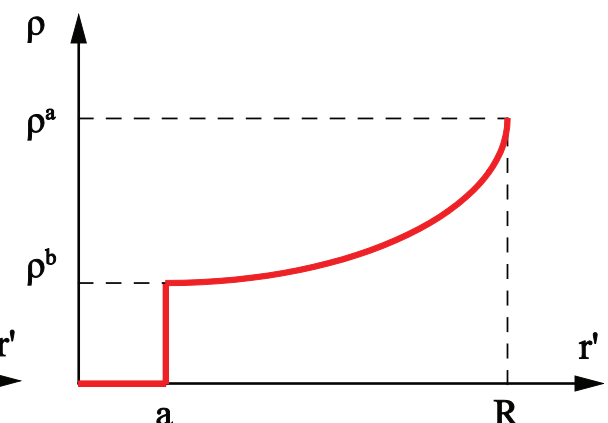

(b)

Figure 8. The profile of a wedge described by exponential functions. (a) The wedge width along the radius during the charging process $(m>0, n>0)$. (b) Dislocation distribution along the radius. Here $\rho^{a}=\frac{1}{2 \pi a} m e^{n^{a}}, \rho^{b}=\frac{1}{2 \pi a} m e^{n}$, and $w^{a}=\frac{m R}{n}\left(e^{n}-e^{n^{a}}\right)$.

$$
\begin{aligned}
& \sigma_{r r}=\frac{G m}{2 \pi(1-\nu)} \frac{1}{n^{2}} \frac{1}{\left(\frac{r}{R}\right)^{2}}\left[\left(n \frac{r}{R}-1\right) e^{\frac{n r}{R}}-\left(n \frac{a}{R}-1\right) e^{\frac{n a}{R}}\right]+ \\
& \frac{G m}{2 \pi(1-\nu)} \int_{\frac{n r}{R}}^{n} g^{-1} e^{g} d g+c(R-a) \\
& c=-\frac{G m}{2 \pi(1-\nu)} \frac{1}{n^{2}} \frac{1}{R-a}\left[(n-1) e^{n}-\left(n \frac{a}{R}-1\right) e^{\frac{n a}{R}}\right]
\end{aligned}
$$

In order to obtain the integral, we may use the Taylor expansion of $e^{g}$ with

$$
e^{g}=\sum_{i=0}^{\infty} \frac{g^{i}}{k !}
$$

Hence the explicit expressions of the two stress components are, respectively, obtained as

$$
\begin{aligned}
\sigma_{\theta \theta}= & -\frac{G m}{2 \pi(1-\nu)} \frac{1}{n^{2}} \frac{1}{\left(\frac{r}{R}\right)^{2}}\left[\left(n \frac{r}{R}-1\right) e^{\frac{n r}{R}}-\left(n \frac{a}{R}-1\right) e^{\frac{n a}{R}}\right]+ \\
& \frac{G m}{2 \pi(1-\nu)}\left\{\ln \frac{R}{r}+\sum_{i=1}^{\infty}\left[\frac{n^{i}}{i \cdot i !}\left(1-\frac{r^{i}}{R^{i}}\right)\right]\right\}+c(R-a) \\
\sigma_{r r}= & \frac{G m}{2 \pi(1-\nu)} \frac{1}{n^{2}} \frac{1}{\left(\frac{r}{R}\right)^{2}}\left[\left(n \frac{r}{R}-1\right) e^{\frac{n r}{R}}-\left(n \frac{a}{R}-1\right) e^{\frac{n a}{R}}\right]+ \\
& \frac{G m}{2 \pi(1-\nu)}\left\{\ln \frac{R}{r}+\sum_{i=1}^{\infty}\left[\frac{n^{i}}{i \cdot i !}\left(1-\frac{r^{i}}{R^{i}}\right)\right]\right\}+c(R-a) \\
c= & -\frac{G m}{2 \pi(1-\nu)} \frac{1}{n^{2}} \frac{1}{R-a}\left[(n-1) e^{n}-\left(n \frac{a}{R}-1\right) e^{\frac{n a}{R}}\right]
\end{aligned}
$$

We show in Figure 9 the normalized stresses (by $\sigma_{0}$ with $\sigma_{0}=\frac{G m}{2 \pi(1-\nu)}$ ) as a function of radius. Note that $m$ is a parameter only appears in $\sigma_{0}$ and we take $n=2$ here. For $m>0$, there is an increasing dislocation density along the radius direction (see Figure 3(b)), The stresses are similar to those induced by the wedge shown in Figure 3. The hoop stress is tensile in the inner core but compressive in the outer shell (Figure 9(a)). The hoop stress and the radial stress decrease when we increase the $a / R$ ratio, as seen in 


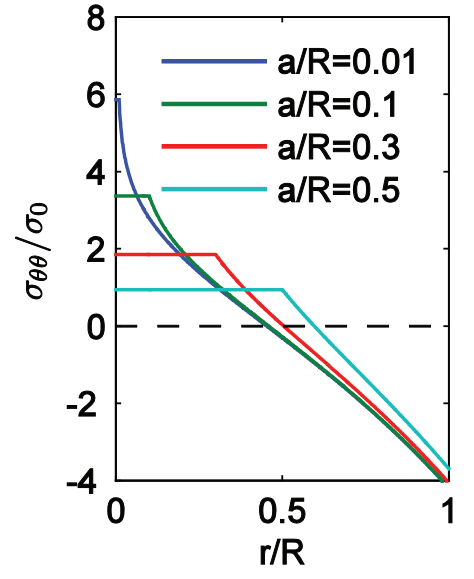

(a)

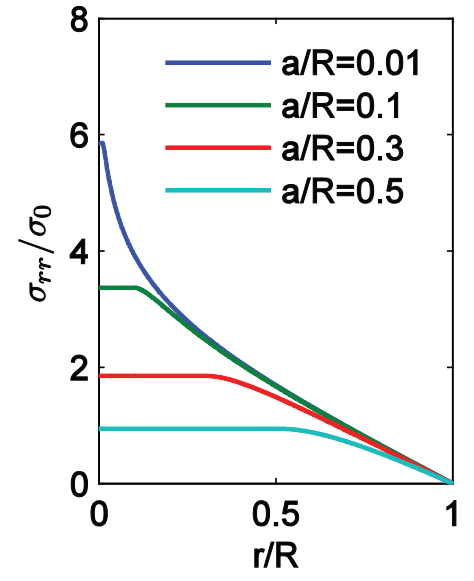

(b)

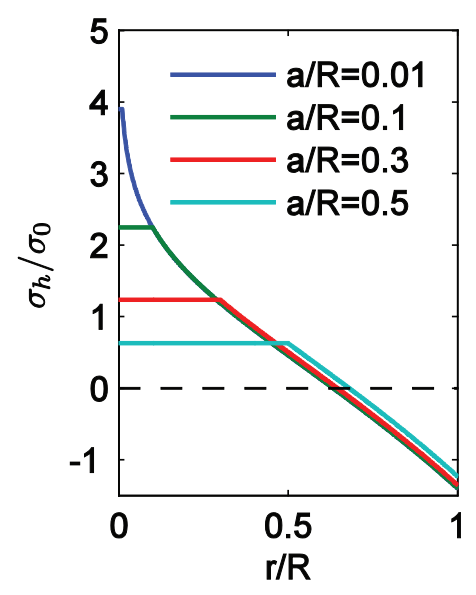

(c)

Figure 9. The stress with exponentially increasing dislocation distribution in different inner-outer diameter ratio (in equation (2I), $m>0, n=2$ ). (a) The evolution of hoop stress as a function of radius. (b) The radial stress. (c) The hydrostatic tensile stress.

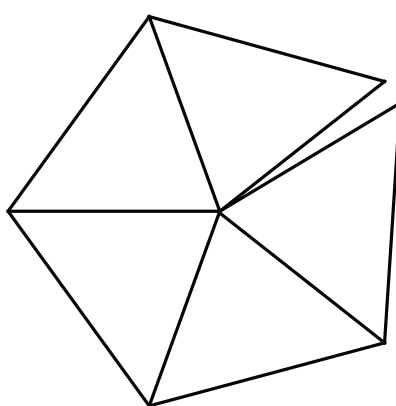

(a)

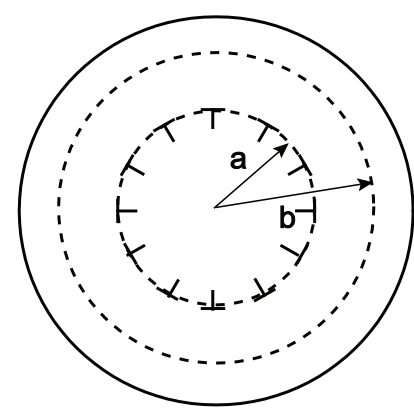

(b)

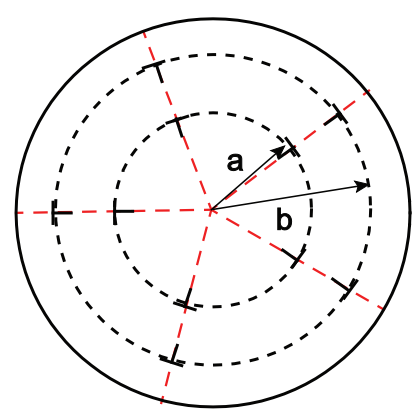

(c)

Figure 10. (a) Sketch of the penta-twinned nanostructure with an angular deficiency. (b) The penta-twinned nanostructure with uniform radial dislocations. (c) The penta-twinned nanostructure with discrete dislocations along the twin boundary.

Figure 9(a) and (b). If we consider the opposite case and take the fully charged state as 'stress-free' state, and then start to discharge the wire, the wedge profile may take the negative value of that seen in Figure 8(a), with the corresponding negative dislocation distribution seen in Figure 8(b). The stresses, if normalized by $\sigma_{0}$, would change signs as $m<0$. In that circumstance, both the hoop stress and the hydrostatic stress are tensile. High tensile stress in the wire surface can then introduce surface cracking.

\section{Discussion and conclusions}

With growing interest in understanding the volume expansion and subsequent stress-induced failure in both higher capacity lithium-ion batteries $[1-3,40]$ and material processing, analytical tools for stress analysis are desired. Letting the outer radius $R \rightarrow \infty$, the stress solutions given here could be used to obtain the stress field introduced by the insertion of wedges in an elastic half-space.

We demonstrate next for stress analysis in penta-twinned NWs. We consider a penta-twinned NW which carries an intrinsic stress field due to the angular elastic misfit with an angular deficiency of $7.35^{\circ}$ [41]. Figure 10 shows the sketch of a penta-twinned NW. We may deduce the residual stress field of penta-twinned NWs by either assuming uniform radial dislocations (Figure 10(b)) or discrete dislocations only distributing in the twin boundary (Figure 10(c)). For the uniform dislocation distribution case, it is equivalent to the disclination model we discussed in Section 3.1. From equation (8), we obtain 


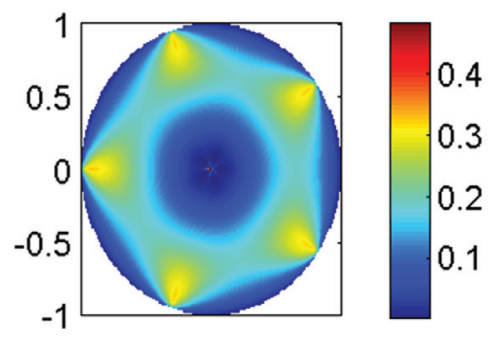

(a)

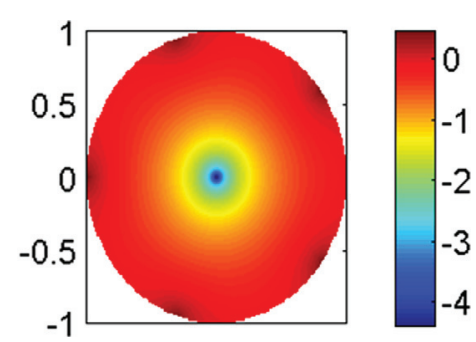

(b)

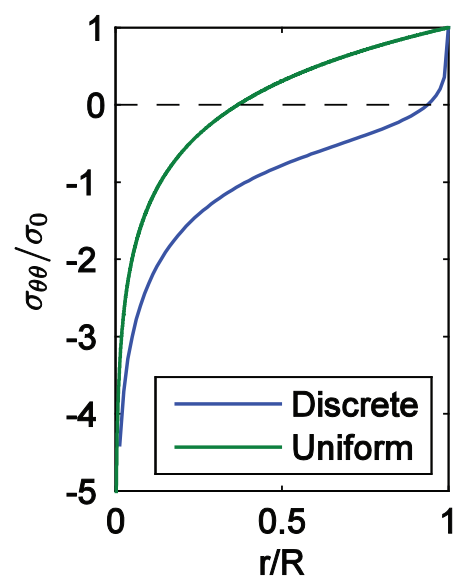

(c)

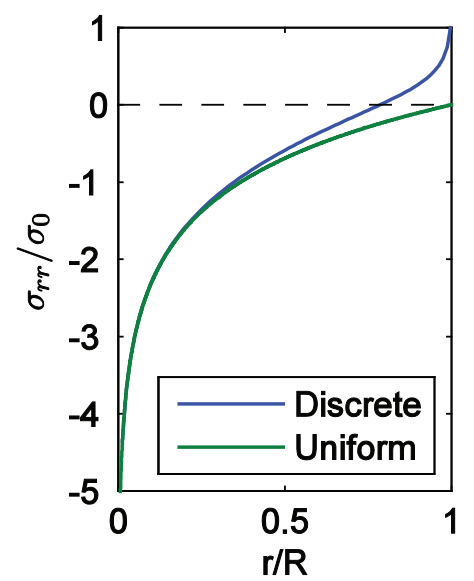

(d)

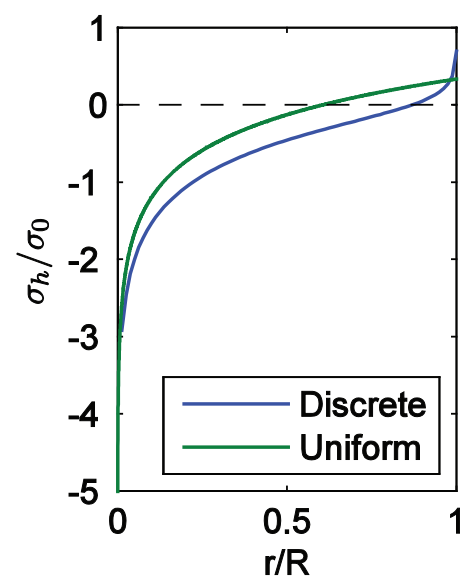

(e)

Figure I I. Residual stress field normalized by $\sigma_{0}$ in the penta-twinned nanostructure. Contours of the (a) von Mises and (b) the hydrostatic stress from discrete dislocation distributed in twin boundaries. (c) $\sigma_{\theta \theta}$, (d) $\sigma_{r r}$, and (e) hydrostatic stress as a function of $r / R, r$ being the radial distance and $R$ the radius of NWs, where $\sigma_{h}$ is the hydrostatic stress.

the residual stress at $r=R$ in the wire (in cylindrical coordinate) as $\sigma_{\theta \theta}=\frac{G \omega^{a}}{2 \pi(1-\nu)}$, where $\omega^{a}=-7.35^{\circ} \times \frac{2 \pi}{360^{\circ}}=-0.128$ is the misfit angular.

The stress field introduced by a discrete dislocation in twin boundaries is numerically solved. We can see from Figure 11 that the von Mises stress exhibits a quinquefoliate-like distribution with the local maximum in twin boundaries and the local minimum at the bisecting line of two neighboring boundaries. For the discrete dislocation case, the highest tensile stress appears at the junction of free surface and twin boundaries. Those observations are in consistent with results from atomistic simulations [42]. Even for the uniform radial expansion cases, high level tensile stress builds up near the surface. For penta-twinned silver NWs, $G=30 \mathrm{GPa}$ and $\nu=0.37$, we have $\sigma_{\theta \theta}=\sigma_{0} \approx 970 \mathrm{MPa}$. Such a high tensile hoop stress at the surface limits the radius of penta-twinned silver NWs, as NWs of large radius may be unable to sustain such a high stress without yield.

Combining with the elastic solution when dislocations are uniformly distributed in the hoop direction [38] and the disclination solution in an infinitely long isotropic cylinder [22,34], we derive the stresses in long wires subjected to arbitrary volume expansion/shrinkage. It has been demonstrated from our results that the peak stress may occur in the core of the wire or at its surface, depending on the detailed profile of volume expansion. While it is generally recognized that the stress field by a single dislocation is long-range, the uniformly distributed dislocation array in a ring is short-range in nature, as seen in Figure 2. It is noted that we neglect the material's property change during material flow. It has been identified that the concentration of lithium ions in $\mathrm{Si}$, for example, may not only change the elastic properties of the structure [43], but also alters its plastic properties like yielding strength [44] and brittleductile behavior $[45,46]$. Furthermore, we note that the elastic theory may only be applicable to structures with small volume change. The solution in NWs with a given volume expansion profile in the radial direction neglects the coupling between stress and diffusion, which may be important for transient 
stress analysis $[20,27,28,47]$. In future work, development of theoretical models may be refined to include the aforementioned mechanisms and time-dependent stress fields.

\section{Acknowledgement}

The authors are grateful to Professor Yang-Tse Cheng at the University of Kentucky for fruitful discussions.

\section{Funding}

This work was supported by the National Natural Science Foundation of China (grant number 11425211).

\section{References}

[1] Aricò, AS, Bruce, P, Scrosati, B, et al. Nanostructured materials for advanced energy conversion and storage devices. Nat Mater 2005; 4: 366-377.

[2] Armand, M, and Tarascon, JM. Building better batteries. Nature 2008; 451: 652-657.

[3] Long, JW, Dunn, B, Rolison, DR, et al. Three-dimensional battery architectures. Chemical Reviews, 2004; 104: 4463-4492.

[4] Kasavajjula, U, Wang, C, and Appleby, AJ. Nano- and bulk-silicon-based insertion anodes for lithium-ion secondary cells. J Power Sources 2007; 163: 1003-1039.

[5] Piper, DM, Evans, T, Xu, S, et al. Optimized silicon electrode architecture, interface, and microgeometry for nextgeneration lithium-ion batteries. Adv Mater 2016; 28: 188-193.

[6] Zhang, Q, Xiao, X, Zhou, W, et al. Toward high cycle efficiency of silicon-based negative electrodes by designing the solid electrolyte interphase. Adv Energy Mater 2015; 5: 1401398.

[7] Armstrong, G, Armstrong, AR, Bruce, PG, et al. $\mathrm{TiO}_{2}$ (B) nanowires as an improved anode material for lithium-Ion batteries containing $\mathrm{LiFePO}_{4}$ or $\mathrm{LiNi}_{0.5} \mathrm{Mn}_{1.5} \mathrm{O}_{4}$ cathodes and a polymer electrolyte. Adv Mater 2006; 18: 2597-2600.

[8] Chan, CK, Zhang, XF, and Cui, Y. High capacity Li ion battery anodes using Ge nanowires. Nano Lett 2007; 8: 307-309.

[9] Chen, $\mathrm{LB}, \mathrm{Lu}, \mathrm{N}, \mathrm{Xu}, \mathrm{CM}$, et al. Electrochemical performance of polycrystalline $\mathrm{CuO}$ nanowires as anode material for $\mathrm{Li}$ ion batteries. Electrochim Acta 2009; 54: 4198-4201.

[10] Chon, MJ, Sethuraman, VA, McCormick, A, et al. Real-time measurement of stress and damage evolution during initial lithiation of crystalline silicon. Phys Rev Lett 2011; 104: 045503.

[11] Cui, LF, Yang, Y, Hsu, CM, et al. Carbon-silicon core-shell nanowires as high capacity electrode for lithium ion batteries. Nano Lett 2009; 9: 3370-3374.

[12] Hosono, E, Kudo, T, Honma, I, et al. Synthesis of single crystalline spinel $\mathrm{LiMn}_{2} \mathrm{O}_{4}$ nanowires for a lithium ion battery with high power density. Nano Lett. 2008; 9: 1045-1051.

[13] Hosono, E, Matsuda, $\mathrm{H}$, Honma, I, et al. Synthesis of single crystalline electro-conductive $\mathrm{Na}_{0.44} \mathrm{MnO}_{2}$ nanowires with high aspect ratio for the fast charge-discharge Li ion battery. J Power Sources 2008; 182: 349-352.

[14] Huang, JY, Zhong, L, Wang, CM, et al. In situ observation of the electrochemical lithiation of a single $\mathrm{SnO}_{2}$ nanowire electrode. Science 2010; 330: 1515-1520.

[15] Kim, H, and Cho, J. Superior lithium electroactive mesoporous Si@ carbon core-shell nanowires for lithium battery anode material. Nano Lett 2008; 8: 3688-3691.

[16] Lee, SW, McDowell, MT, Berla, LA, et al. Fracture of crystalline silicon nanopillars during electrochemical lithium insertion. Proc Natl Acad Sci 2012; 109: 4080-4085.

[17] Meduri, P, Pendyala, C, Kumar, V, et al. Hybrid tin oxide nanowires as stable and high capacity anodes for Li-ion batteries. Nano Lett 2009; 9: 612-616.

[18] Seo, MH, Park, M, Lee, KT, et al. High performance Ge nanowire anode sheathed with carbon for lithium rechargeable batteries. Energy Environ Sci 2011; 4:425-428.

[19] Ryu, LSW, Gao, HJ, Cui, Y, et al. Microscopic model for fracture of crystalline Si nanopillars during lithiation. J. Power Sources, 2014; 255: 274-282.

[20] Bower, AF, Guduru, PR, and Sethuraman, VA. A finite strain model of stress, diffusion, plastic flow and electrochemical reactions in a lithium-ion half-cell. J Mech Phys Solids 2011; 59: 804-828.

[21] Christensen, J, and Newman, J. A mathematical model of stress generation and fracture in lithium manganese oxide. J Electrochem Soc 2006; 153: A1019-A1030.

[22] Christensen, J, and Newman, J. Stress generation and fracture in lithium insertion materials. $J$ Solid State Electrochem 2006; 10: 293-319.

[23] Di Leo, CV, Rejovitzky, E, and Anand, L. A Cahn-Hilliard-type phase-field theory for species diffusion coupled with large elastic deformations: application to phase-separating Li-ion electrode materials. J Mech Phys Solids 2014; 70: 1-29.

[24] Deshpande, R, Cheng, YT, and Verbrugge, MW. Modeling diffusion-induced stress in nanowire electrode structures. J Power Sources 2010; , 195: 5081-5088. 
[25] Pan, J, Zhang, Q, Li, J, et al. Effects of stress on lithium transport in amorphous silicon electrodes for lithium-ion batteries. Nano Energy 2015; 133: 192-199.

[26] Prussin, S. Generation and distribution of dislocations by solute diffusion. J Appl Phys 1961; 32: $1876-1881$.

[27] Zhang, X, Sastry, AM, and Shyy, W. Intercalation-induced stress and heat generation within single lithium-ion battery cathode particles. J Electrochem Soc 2008; 155: A542-A552.

[28] Zhang, X, Shyy, W, and Sastry, AM. Numerical simulation of intercalation-induced stress in Li-ion battery electrode particles. J Electrochem Soc 2007; 154: A910-A916.

[29] Cheng, YT, and Verbrugge, MW. Evolution of stress within a spherical insertion electrode particle under potentiostatic and galvanostatic operation. J Power Sources 2009; 190: 453-460.

[30] Cheng, YT, and Verbrugge, MW. Diffusion-induced stress, interfacial charge transfer, and criteria for avoiding crack initiation of electrode particles. J Electrochem Soc 2010; 157: A508-A516.

[31] Bower, AF, Guduru, PR, and Chason, E. Analytical solutions for composition and stress in spherical elastic-plastic lithium-ion electrode particles containing a propagating phase boundary. Int J Solids Struct 2015; 69: 328-342.

[32] Liu, X.H, Zheng, H, Zhong, L, et al. Anisotropic swelling and fracture of silicon nanowires during lithiation. Nano Lett 2011; 11: 3312-3318.

[33] Harris, SJ, Deshpande, RD, Qi, Y, et al. Mesopores inside electrode particles can change the Li-ion transport mechanism and diffusion-induced stress. J Mater Res 2010; 25: 1433-1440.

[34] Volterra, V. Sur l'équilibre des corps élastiques multiplement connexes. Ann Scient Ec Norm Sup 1907; 24: $400-517$.

[35] Eshelby, J. A simple derivation of the elastic field of an edge dislocation. Br J Appl Phys 1966; 17: 1131-1135.

[36] Obrovac, MN, and Christensen, L. Structural changes in silicon anodes during lithium insertion/extraction. Electrochem Solid-State Lett 2004; 7: A93-A96.

[37] Hatchard, TD, and Dahn, JR. In situ XRD and electrochemical study of the reaction of lithium with amorphous silicon. $J$ Electrochem Soc 2004; 151: A838-A842.

[38] Weertman, J. Dislocation based fracture mechanics. Singapore: World Scientific Publishing Co Ltd, 1996.

[39] De Wit, R. Partial disclinations. J Phys C Solid State Phys 1972; 5: 529-534.

[40] Sun, K, Wei, TS, Ahn, BY, et al. 3D printing of interdigitated Li-ion microbattery architectures. Adv Mater 2013; 25: 4539-4543.

[41] Qin, QQ, Yin, S, Cheng, GM, et al. Recoverable plasticity in penta-twinned metallic nanowires governed by dislocation nucleation and retraction. Nat Commun 2015; 6: 5983.

[42] Wu, JY, Nagao, S, He, JY, et al. Role of five-fold twin boundary on the enhanced mechanical properties of fcc Fe nanowires. Nano Lett 2011; 11: 5264-5273.

[43] Song, T, Xia, J, Lee, JH, et al. Arrays of sealed silicon nanotubes as anodes for lithium ion batteries. Nano Lett 2010; 10: 1710-1716.

[44] Zhao, K, Wang, WL, Gregoire, J, et al. Lithium-assisted plastic deformation of silicon electrodes in lithium-ion batteries: a first-principles theoretical study. Nano Lett 2011; 11: 2962-2967.

[45] Ding, B, Li, X, Zhang, X, et al. Brittle versus ductile fracture mechanism transition in amorphous lithiated silicon: From intrinsic nanoscale cavitation to shear banding. Nano Energy 2015; 18: 89-96.

[46] Xia, SM, Gao, YF, Bower, AF, et al. Delamination mechanism maps for a strong elastic coating on an elastic-plastic substrate subjected to contact loading. Int J Solids Struct 2007; 44: 3685-3699.

[47] Bower, AF, and Guduru, PR. A simple finite element model of diffusion, finite deformation, plasticity and fracture in lithium ion insertion electrode materials. Modell Simul Mater Sci Eng 2012; 20: 45004-45023. 